\title{
Geomagnetic Storms' Influence on Intercity Railway Track Circuit
}

\author{
Xuecheng Qian ${ }^{1,2} \cdot$ Hao $\operatorname{Tian}^{2} \cdot$ Yanlin $\mathrm{Yin}^{3} \cdot$ Yang $\mathrm{Li}^{2} \cdot$ Mingguang Liu ${ }^{2}$. \\ Zeming Jiang ${ }^{4}$
}

Received: 25 March 2016/Revised: 28 June 2016/ Accepted: 29 June 2016/Published online: 8 July 2016

(c) The Author(s) 2016. This article is published with open access at Springerlink.com

\begin{abstract}
Geomagnetic storms can cause earth surface potentials (ESPs) in the ground. ESP produces geomagnetically induced current (GIC) in a loop which is made up by rails and ground. If GIC flows into intercity railway track circuit, it will threaten the normal operation of track circuit. The reason that geomagnetic storms invade the track circuit was analyzed, and then how GIC affects the characteristics of choke transformers, was found. The calculation method of GIC according to a simulation based on the GIC flow path in the track circuit was built. Electromagnetic system model of choke transformer and track relay were designed in Maxwell
\end{abstract}

Xuecheng Qian

13121456@bjtu.edu.cn

Hao Tian

14121462@bjtu.edu.cn

Yanlin Yin

13125964@bjtu.edu.cn

Yang Li

14125977@bjtu.edu.cn

Mingguang Liu

mgliu@bjtu.edu.cn

Zeming Jiang

zemingji@usc.edu

1 State Grid Changzhou Power Supply Company, Changzhou, Jiangsu, China

2 School of Electrical Engineering, Beijing Jiaotong University, Haidian District, West 504, Beijing, China

3 State Grid Jinchen Power Supply Company, Jinchen, Shanxi, China

4 School of Electrical Engineering, University of Southern California, Los Angeles, USA

Editor: Baoming Han software to get the magnetic flux density distribution of them. The results show that GIC values of $2.4 \mathrm{~A}$ can bring about serious direct current bias of choke transformers and cause the terminal voltage of track relay to decrease, resulting in relay malfunction. The numerical calculation results show that geomagnetic storms will interfere with intercity railway track circuit, which is in accordance with some phenomenon happened in some rail tracks. This method proves geomagnetic storms' influence on intercity railway track circuit and attentions should be paid to this influence.

Keywords Geomagnetic storms · Intercity railway · Track circuit - Choke transformer · Track relay

\section{Background}

When the solar actives intensely, a large number of plasma jets out with the velocity of $300-1000 \mathrm{~km} / \mathrm{s}$ in the interplanetary space near the earth, so geomagnetic storms are formed. Then the surface magnetic field together with electric potential change obviously. Huge geomagnetic storms follow a 10- to 11-year sunspot cycle and geomagnetic storms can produce different surface magnetic fields and potential in different geographical locations [1], inducing the earth surface potentials (ESPs).

Track circuit is an important part of intercity railway signaling system, playing a vital role in urban rail traffic order and safety. In May 1921, the US suffered geomagnetic storm interference in the railway system. In Sweden, when strong geomagnetic storms happened, the signal light of track circuit "flashed red" in July 1982. In 1989 and 2000-2005, strong geomagnetic storms caused the problem that northern midlatitudes in Russian and Gorky electrification railway section were observed much operation 
abnormity in the track signal system. These phenomena were found by Liu [2], Wik et al. [3], and Beloa et al. [4]. Molinski [5] and Gummow and Eng [6] found geomagnetic storms can influence long conductors on the ground surface. Mendes et al. [7] used instruments to detect geomagnetic disturbance. Tessier and Simard [8] realized ESP can be produced by magnetic storms. The geometry of the geomagnetic field causes a westward ring current [9]. Oyedokun et al. [10] made a calculation of geomagnetically induced currents (GICs) in transmission networks. These articles reported and found geomagnetic storms can have disadvantages of electrical system on the ground.

How geomagnetic storms affected domestic long-distance power transmission systems and pipelines has already been observed and discussed [11-13]. Because rails are very long and exposed in the field as long conductors, they can be analogous to transmission lines, oil pipelines, and other long conductors, so we could infer that geomagnetic storms would also affect the track circuit, and it is necessary to study this effect scientifically.

$25 \mathrm{~Hz}$ phase-sensitive track circuit is one of the intercity railway infrastructures. Intercity railway communication and control systems often work in sensitive status, because they adopt microelectronics and computer systems. The geomagnetic storms will easily affect the track circuit and signal equipment [14]. Therefore, the new problem that geomagnetic storms interfere with the track circuit is worth studied.

\section{The Functions and Composition of Track Circuit}

Track circuit contains rails and types of equipments connected to its beginning and end terminal, used to detect whether there is a train in the railway. Track circuit sends, transmits, and receives signals, displaying the signal light reflecting the state of railway operation. It is equipment essential to ensure the safety of railway operation. $25 \mathrm{~Hz}$ phase-sensitive track circuit is composed of sending electricity side, receiving electricity side and rails as the signal channel. The main equipments involved in the track circuit are choke transformers $\left(\mathrm{BE}_{25}\right)$, track transformers $\left(\mathrm{BG}_{25}\right)$, track relay (JRJC), and equivalent impedance of rails. Different sections of the track circuit are segregated with electrical isolation. All parts are illustrated in Fig. 1.

\section{The Mechanism of GIC Affecting the Intercity Railway Track Circuit}

Geomagnetic storms can induce ESP in the earth surface, and then ESP, rails, and ground form a circuit loop, so GIC is produced in the loop. Choke transformers are installed on

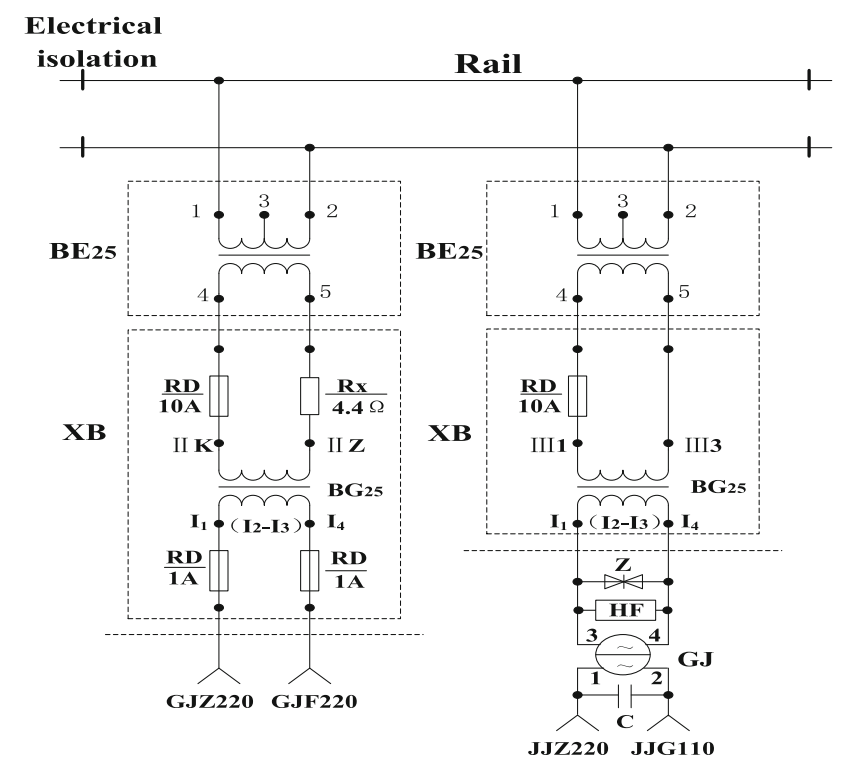

Fig. 1 Composition of $25 \mathrm{~Hz}$ phase-sensitive track circuit

both sides of electrical isolation on rails and the GIC circulation path within two adjacent electrical isolations is rails, choke transformers, and ground. The GIC circulation path is illustrated in Fig. 2. As a result, GIC invades into the choke transformers. The frequency of GIC is $0.001-0.1 \mathrm{~Hz}$, so it can be seen as quasi direct current (DC) [15]. GIC transmits in the same direction on two different rails. If two rails are completely symmetrical, GIC will be equal in circulation on the two rails and the influence of GIC on the choke transformers will offset due to the symmetry of two rails, so there is no DC bias of choke transformers. But the impedance of two rails may not be equal in the actual project. For example, the lengths of two rails are different in inner and outer corners; two rails are in different geological and damp conditions, resulting in the different resistors of track bed. In addition, the manufacturing process that two 8 turns of coils in the choke transformers are asymmetric, etc. These factors will cause different GIC to flow on the two rails, resulting in DC bias

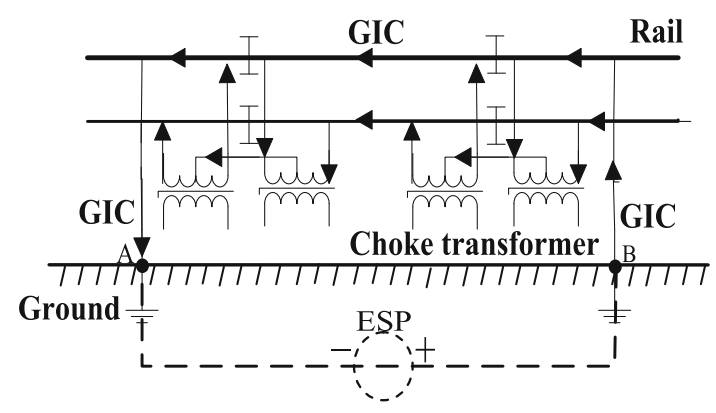

Fig. 2 Schematic diagram of GIC generated and its flow path on the rails 
of the choke transformers and causing core saturation of choke transformers.

Apart from carrying vehicles, rails play a role as a carrier for signal current path and it is not affected by GIC. The cross-sectional area of rails is large, so the equivalent impedance of rails is small and the value of GIC will be bigger at the same value of ESP. Such a huge current flows through rails will have a serious impact on the track circuit. Choke transformers in the track circuit are easily affected by GIC and track relay is weakly able to resist electromagnetic interference [14], so the paper mainly analyzes how GIC affects the choke transformers and track relay in $25 \mathrm{~Hz}$ phase-sensitive track circuit.

\section{GIC's influences on Choke Transformers}

Transformers are electromagnetic devices, and their core has saturated characteristic. During normal operation, choke transformers work in the linear situation, and their operating point is located in the curve "OA" section of core magnetization. Working characteristic curve of choke transformers is shown in Fig. 3b. When transformers work in rated operation, they work at "A point" in Fig. 3b, making full use of main magnetic flux and ferromagnetic materials. If GIC flows into transformer windings, it can produce DC flux $\phi_{0}$ in the choke transformers. $\phi_{0}$ superimposes with the main flux $\phi$, so the flux curve shifts upward, as is shown in "Curve 1 " of Fig. 3a, resulting that the flux magnitude in half cycle of wave increases sharply. However, the other side of wave decreases sharply, as is shown in "Curve 2" of Fig. 3c. It will cause choke transformers to operate in the nonlinear magnetization curve, resulting in waveform distortion of transformer magnetizing current.

The traction windings of choke transformers are connected on both sides of the electrical isolation on rails. The midpoint of the two traction windings in choke transformers is connected by cable, supplying path for traction current. The flow path of GIC and traction current is consistent. What is different is that GIC is seen as quasi DC, but the frequency of traction current is $50 \mathrm{~Hz}$, so two kinds of currents are independent of each other. When the GIC on two rails is

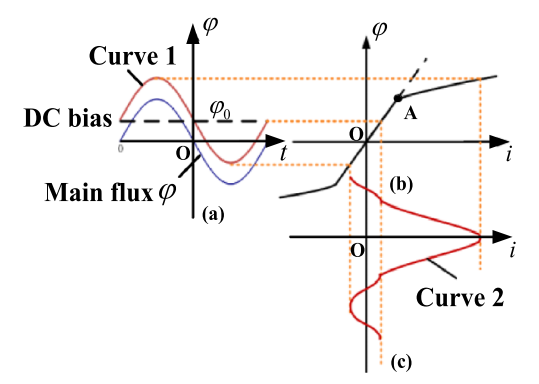

Fig. 3 DC bias schematic diagram of choke transformer asymmetric, the traction windings of choke transformers will generate the difference between two currents and interfered voltage will be formed. Interfered voltage is coupled to the signal coil; together with the reason that gap of choke transformer core is small, the more degree of asymmetry the GIC is, the more the interfered voltage will be [16]. As a result, due to the above-mentioned factors, GIC is easier to cause core saturation and DC bias of choke transformers, resulting in interference of track circuit equipments.

\section{Calculation Method of GIC and Results}

When geomagnetic storms happen, ESP is constantly changing as time changes, and we use the peak value of ESP to calculate GIC. Since ESP is different between consequent associated adjacent track circuits, consider that the region of certain track circuit is limited, so that the ground electric field strength $\vec{E}$ can be consistent. This paper puts ESP on rails between two ground points. The contact resistance of rail to ground is manly composed of leakage current resistance by rails, ballast, and roadbed. Because GIC is seen as quasi DC, capacitance and inductance along the line can be neglected. The filter of track circuit has no impact on the DC, so this paper ignores the filter link in order to simplify the calculation. The actual equivalent circuit of track circuit contains resistor and voltage source, and we use four-node network as an example. The equivalent model to calculate GIC is shown in Fig. 4, where $U_{1}-U_{3}$ represent, respectively, the equivalent voltage sources acting on different sections of unit length of rails as ground-induced electric field, $R_{1}-R_{3}$ represent, respectively, the different sections of unit resistance of rails, and $R_{01}-R_{04}$ represent, respectively, the different sections of rail-to-ground contact resistance.

The calculation of voltage is

$U_{i j}=\int_{i}^{j} \vec{E} \cdot \mathrm{d} \vec{l}$.

In dealing with Eq. (1), if the long conductor is divided into $N$ segments, a short differential segments $[i, j]$ included

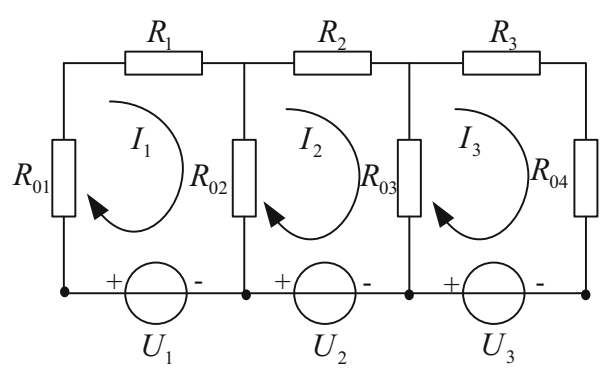

Fig. 4 Equivalent model of track circuit to calculate GIC (four-note network) 
in the long conductor can be approximately seen as a straight line. So a short length of the ground EMF can also be seen as a uniform potential. The direction of earth's magnetic field is always from the North Pole to the South Pole. This paper sets north direction for the $X$-axis Cartesian coordinate system, east direction for the $Y$-axis Cartesian coordinate system, and $Z$-axis direction perpendicular to the ground (the direction of induced current). Consider the short differential segments $[i$, $j$ ] of long conductor to be nonlinear, wherein the induced electric field direction $\vec{E}$ at any point is inconsistent with long conductor. $E_{x}$ and $E_{y}$ reflect the north and east directions of the component, respectively, of the ground electric field in the long conductor. $\theta$ is the angle between a short differential $[i, j]$ in the long conductors with $Y$-axis (east-west), and the total equivalent voltage source in a short differential $[i, j]$ in the long conductors can be calculated using Eq. (2):

$U_{i j}=L_{i j}\left(E_{x} \sin \theta+E_{y} \cos \theta\right)$,

where $L_{i j}$ is the length of a short differential $[i, j]$. The equivalent voltage source of all long conductors is

$U_{\mathrm{AB}}=\sum_{1}^{N} U_{i j}$.

When the scale of ground long conductor is not particularly large or we consider and study the relatively small scope of long conductor, electric field on the ground can be approximately considered as a uniform electric field. We know that Eq. (1) has nothing to do with the path approximately. So the potential equivalent voltage source ESP between long conductors AB [Eq. (3)] in the ground can be simplified to Eq. (4) [17]:

$U_{\mathrm{AB}}=L_{\mathrm{AB}}\left(E_{x} \sin \theta+E_{y} \cos \theta\right)$,

where $L_{\mathrm{AB}}$ is the length of a long conductor $\mathrm{AB}$ and $\theta$ is the angle between the line of $\mathrm{AB}$ and $Y$-axis (east-west).

This paper uses loop current method to calculate the size of GIC on each branch loop, and the above model can be calculated using matrix equation:

$$
\begin{aligned}
& {\left[\begin{array}{ccc}
R_{01}+R_{1}+R_{02} & -R_{02} & 0 \\
-R_{02} & R_{02}+R_{2}+R_{03} & -R_{03} \\
0 & -R_{03} & R_{03}+R_{3}+R_{04}
\end{array}\right]\left[\begin{array}{l}
I_{1} \\
I_{2} \\
I_{3}
\end{array}\right]} \\
& =\left[\begin{array}{c}
U_{1} \\
U_{2} \\
U_{3}
\end{array}\right],
\end{aligned}
$$

where $I_{1}-I_{3}$ represent GIC flows on each of the track circuit, respectively, and by solving Eq. (5), the value of GIC on each of the track circuit can be calculated, and then asymmetric GIC flowing into the choke transformer can also be figured out.
Figure 5 shows an equivalent circuit that GIC flows into traction power supply network. The position of the running train is $S$ kilometers from traction power supply. Referring to the provisions of GB 5016-9, taking $R_{0}=1 \Omega$ as the rail-to-ground leakage resistance and $r_{0}=0.2 \Omega / \mathrm{km}$ as resistance of per unit length of rails.

Pirjola [18] pointed out that when strong geomagnetic storms happen, the maximum value of the induced electric

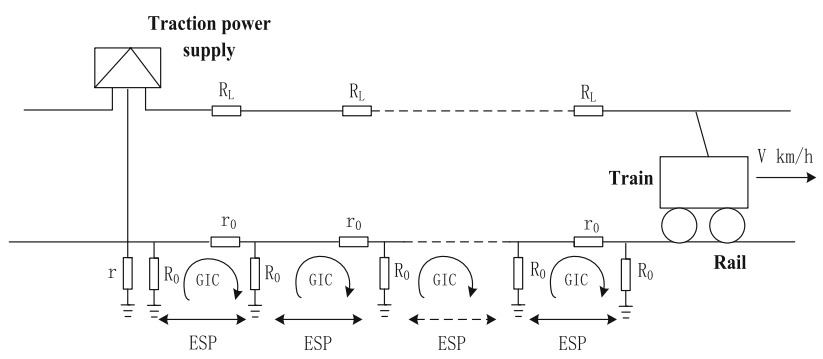

Fig. 5 Equivalent circuit that GIC flows in traction power supply network

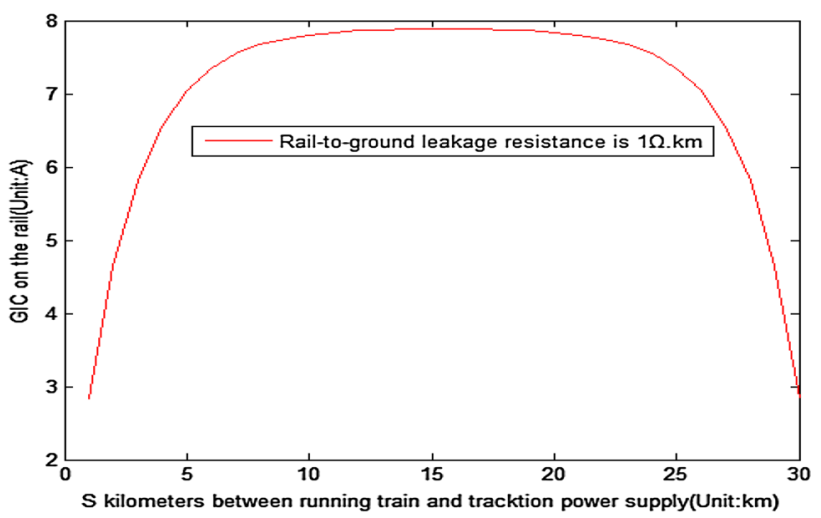

Fig. 6 The relationship between GIC and $S$ (calculated using Matlab)
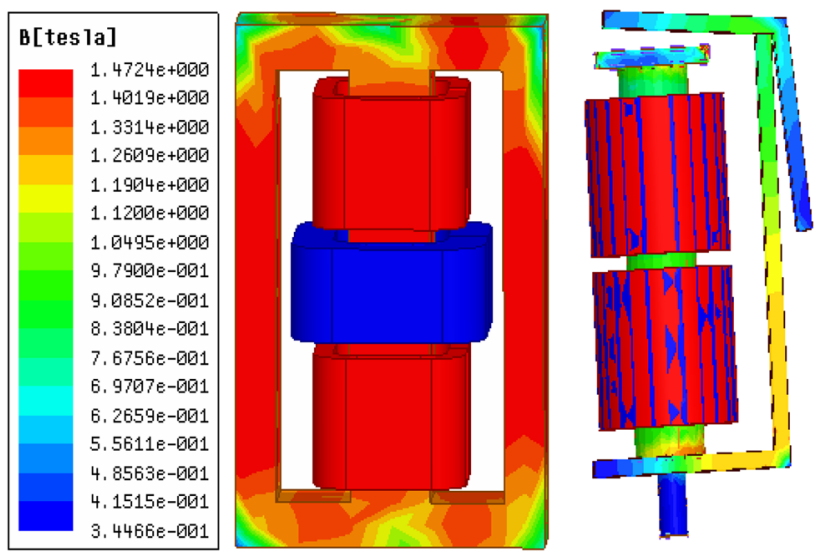

Fig. 7 (GIC = 0 A) the magnetic flux density distribution of choke transformer and track relay 
Fig. 8 The waveform

displaying the terminal voltage of track relay

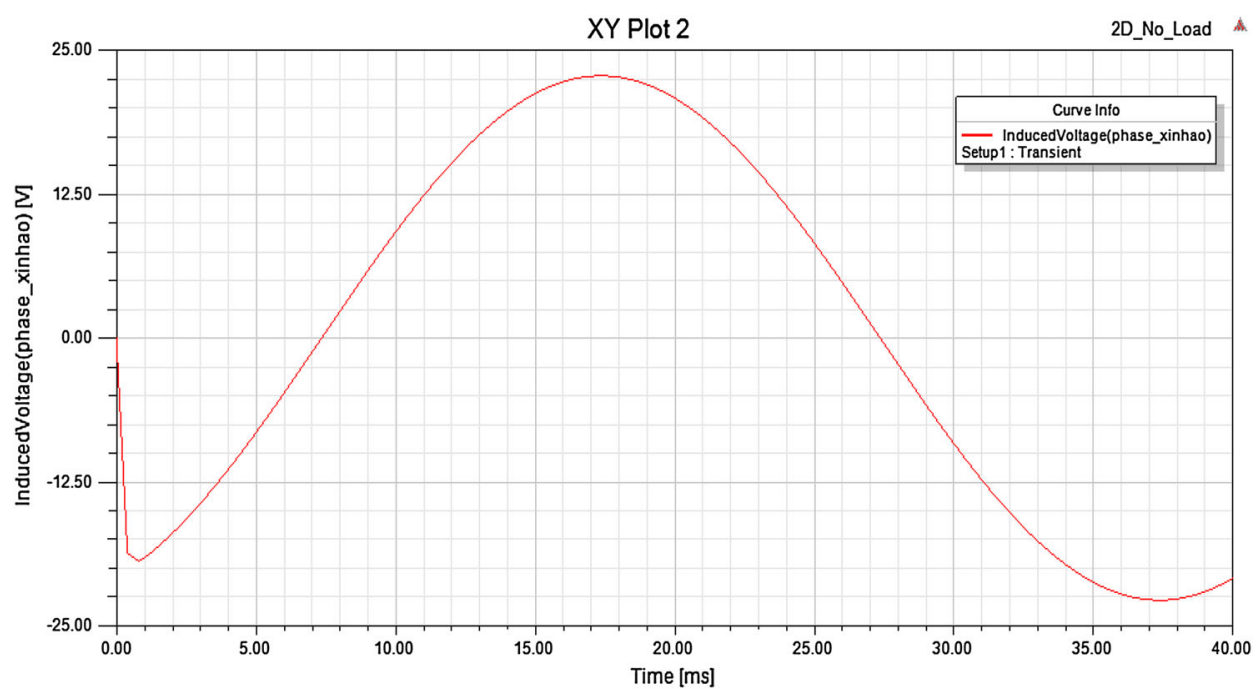

(a) $\mathrm{GIC}=0 \mathrm{~A}$

XY Plot 2

2D_No_Load A

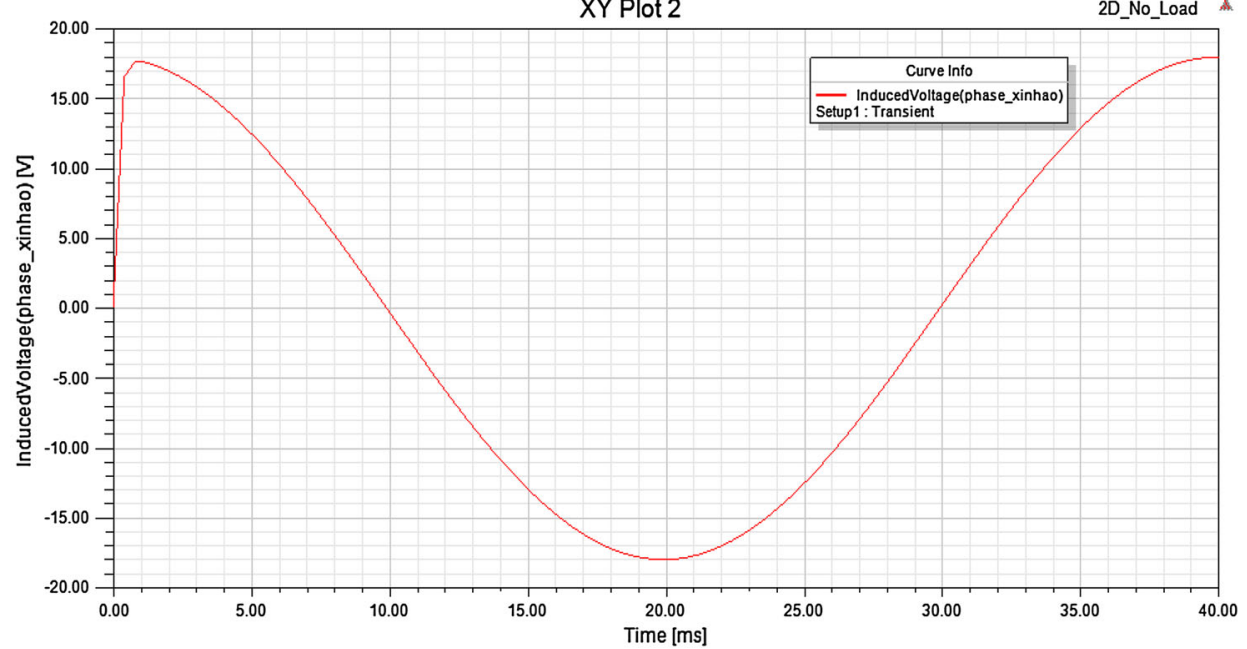

(b) GIC $=0.4 \mathrm{~A}$

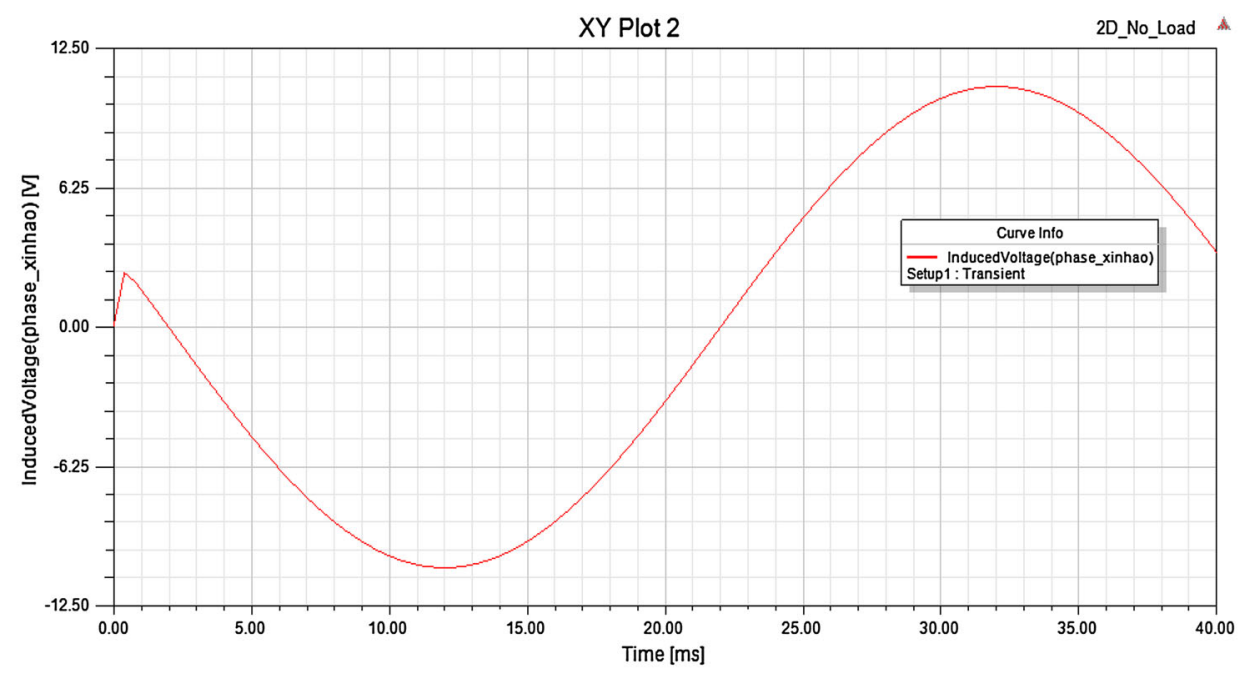

(c) $\mathrm{GIC}=2.4 \mathrm{~A}$ 


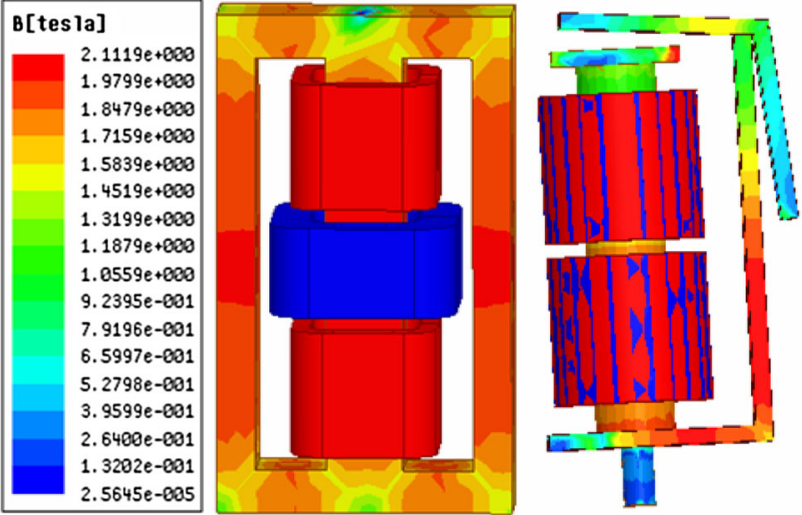

Fig. 9 ( GIC $=2.4 \mathrm{~A})$ the magnetic flux density distribution of choke transformer and track relay

field can reach to $1 \mathrm{~V} / \mathrm{km}$. So this paper sets ESP $=1 \mathrm{~V}$. Consider that the train starts from the traction substation, combined with Eq. (5) using Matlab software to calculate the GIC flowing on rails. The distance from the train to traction power supply is $S$, and the relationship between GIC and $S$ is shown in Fig. 6.

The result shows that when geomagnetic storms happen, the maximum value of GIC on rails is $7.9 \mathrm{~A}$. Assume that the degree of asymmetric current on rails is $5 \%$, so asymmetric GIC generated is $7.9 \times 5 \% \approx 0.4 \mathrm{~A}$. However, at high-latitude area, induced voltage can reach to $6 \mathrm{~V}$ each kilometer when strong storms happen [18]. Asymmetric GIC at this time is six times of 0.4 , which is $2.4 \mathrm{~A}$. As is known, the rated current of choke transformers in track circuit is about 600-1000 A. Working in the normal state, magnetizing current of choke transformers is only $1-5 \%$ of the rated current. Therefore, such a huge asymmetric GIC flows in the track circuit cannot be ignored.

\section{Electromagnetic System Model and Results}

The track coil of choke transformers (BE2-600/25) is wrapped of two 8 turns of wire made by flat steel, while the signal coil wrapped of 48 turns of wire made by round copper. The ratio of primary coil windings against secondary coil windings is $1: 3$. The capacity of choke transformers is $3 \mathrm{KVA}$, and the resistance of primary and secondary coils is $R_{1}=R_{2}=0.2 \Omega$. According to the main technical indicators of $25 \mathrm{~Hz}$ phase-sensitive track circuit: when there is no train on rails, the effective voltage of the terminal of track coil relay (WXJ-25) shall not be less than $15 \mathrm{~V}$, and when the effective voltage falls to $9 \mathrm{~V}$, the contact of the track relay will fall down. The operating current of track relay is $0.039 \mathrm{~A}$.
When there is no train and no GIC on rails, according to Tian [19] and Zhai [20] to select the simulation parameters of choke transformers and track relay, ignoring harmonics and other effects. This paper uses Maxwell software to build a simulation, the magnetic flux density distribution of choke transformer and track relay is shown in Fig. 7. The waveform displaying the terminal voltage of track relay is shown in Fig. 8a. The maximum value of terminal voltage is $22.5 \mathrm{~V}$, so the effective value of it is $\frac{22.5}{\sqrt{2}}=15.9 \mathrm{~V}$; at this time, the contact of track relay is sucked by the armature. When asymmetric GIC values of 0.4 A flows into the primary windings of choke transformers, the simulation result shows that the terminal voltage of track relay decreases to $17.9 \mathrm{~V}$, and the effective value of it is $\frac{17.9}{\sqrt{2}}=$ $12.66 \mathrm{~V}$, as shown in Fig. 8b; at this time, the contact of track relay is still sucked. But at high-latitude area, when asymmetric GIC values of 2.4 A flows into the primary windings of choke transformers, the simulation result shows that the terminal voltage of track relay decreases to $10.63 \mathrm{~V}$, and the effective value of it is $\frac{10.63}{\sqrt{2}}=7.51 \mathrm{~V}$, as is shown in Fig. 8c; at this time the contact of track relay will fall down. The magnetic flux density distribution of choke transformer and track relay is shown in Fig. 9. Because the flux density of silicon steel working in normal operation is about $1.5 \mathrm{~T}$, it can be seen from Fig. 9 that asymmetric GIC values of $2.4 \mathrm{~A}$ flowing into the primary windings of choke transformers can cause serious DC bias.

\section{Conclusions}

In this paper, we presented the composition of $25 \mathrm{~Hz}$ phase-sensitive track circuit and show how GIC flows into track circuit. An equivalent model of track circuit has been used to calculate the value of GIC, and equations can be easily calculated by Matlab software showing values of GIC between traction power supply and the running train. The model of GIC affecting choke transformers and track relay can be simulated and analyzed using Maxwell software. We use the calculated values of GIC to display how GIC influences the terminal voltage of track relay. Flux density distribution of choke transformer and track relay are shown when GIC $=0$ and $2.4 \mathrm{~A}$. We can sum up that if GIC flows into track circuit, it will have serious impact on the intercity railway track circuit. The major conclusions drawn from the study are given below:

- Geomagnetic storms can cause ESPs in the ground. ESP produces GIC in a loop which is made up by rails and ground. 
- GIC induced by geomagnetic storms will cause DC bias and half-cycle saturation of choke transformers in intercity railway track circuit.

- Asymmetric GIC values of $2.4 \mathrm{~A}$ that flows into the primary coil windings of choke transformers will bring about serious DC bias in choke transformers and cause track relay malfunction.

- When we do projects in the field, we shall try to ensure that the impedance parameters of two rails consistent to reduce asymmetrical of rails and ensure the manufacturing process of choke transformer's traction coil symmetrical, avoiding asymmetric GIC flowing on two rails.

Acknowledgments This work is partially supported by the Fundamental Research Funds of the Natural Science Foundation of China (No. 41374189).

Open Access This article is distributed under the terms of the Creative Commons Attribution 4.0 International License (http://crea tivecommons.org/licenses/by/4.0/), which permits unrestricted use, distribution, and reproduction in any medium, provided you give appropriate credit to the original author(s) and the source, provide a link to the Creative Commons license, and indicate if changes were made.

\section{References}

1. Ptitsyna NG, Kasinsky VV, Villoresi G et al (2008) Geomagnetic effects on mid-latitude railways: a statistical study of anomalies in the operation of signaling and train control equipment on the East-Siberian Railway. Adv Space Res 42(9):1510-1514

2. Liu H, Yamamoto M (2011) Weakening of the mid-latitude summer nighttime anomaly during geomagnetic storms. Earth Planets Space 63(4):371-375

3. Wik M, Pirjola R, Lundstedt $\mathrm{H}$ et al (2009) Space weather events in July 1982 and October 2003 and the effects of geomagnetically induced currents on Swedish technical systems. Med Sci Entry Ann Geophys 43(6):1775-1787

4. Beloa V, Aidash SPG, Roshenko EA et al (2005) Influence of the great geomagnetic disturbances on the Northern Railways operating. In: 2nd European space weather week, vol 11, Noordwijk, The Netherlands, p 14-18

5. Molinski TS (2002) Why utilities respect geomagnetically induced currents. J Atmos Sol Terr Phys 64(16):1765-1778
6. Gummow RA, Eng P (2002) GIC effects on pipeline corrosion and corrosion control systems. J Atmos Sol Terr Phys 64(16): 1755-1764

7. Mendes O, Domingues MO, Costa AMD et al (2005) Wavelet analysis applied to magnetograms: singularity detections related to geomagnetic storms. J Atmos Sol Terr Phys 67(17):1827-1836

8. Tessier JL, Simard G (2008) Case study of the impact of a magnetic storm on a hydro-Québec distribution feeder. J Am Geriatr Soc 61(7):1217-1220

9. Shen C, Yang YY, Rong ZJ et al (2014) Direct calculation of the ring current distribution and magnetic structure seen by cluster during geomagnetic storms. J Geophys Res Space Phys 119(4):2458-2465

10. Oyedokun DTO, Simon MN, Gaunt CT (2013) Introduction of a more detailed calculation of geomagnetically induced currents in transmission networks. In: Southern African University's power engineering conference

11. Viljanen A, Pulkkinen A, Pirjola R et al (2006) Recordings of geomagnetically induced currents and a now casting service of the Finnish natural gas pipeline system. Space Weather 4(12):10-28

12. Wu WL, Liu LG, Liu CM (2014) Voltage stability in a longdistance power transmission system impacted by the geoelectric field due to a geomagnetic disturbance. Earth Sci Inform 7(3):173-185

13. Osella A, Favetto A, López E (1998) Currents induced by geomagnetic storms on buried pipelines as a cause of corrosion. J Appl Geophys 38(3):219-233

14. Fan T (2012) Impacts of geomagnetic storms on track circuit. Railw Signal Commun 48(7):6-7 (in Chinese)

15. Wik M, Pirjola R, Lundstedt $\mathrm{H}$ et al (2009) A space weather events in July 1982 and October 2003 and the effects of geomagnetically induced currents on Swedish technical systems. Ann Geophys 27(4):1775-1787

16. Liu Q (2012) Analysis on magnetic saturation of choke transformer and precaution. Xi' an Railw Technol 4(3):20-21 (in Chinese)

17. Qian X (2015) Analysis on how electromagnetic interference of geomagnetic storms affects track circuit. J China Railw Soc 37(5):42-46 (in Chinese)

18. Pirjola R (2007) Calculation of geomagnetically induced currents (GIC) in a high-voltage electric power transmission system and estimation of effects of overhead shield wires on GIC modeling. J Atmos Sol Terr Phys 69(4):1305-1311

19. Tian M (2011) Equivalent circuit of choke transformer. Transformer 48(5):30-32 (in Chinese)

20. Zhai G (2008) Finite element analysis of static characteristics of electromagnetic relay interfered by static magnetic field. Proc CSEE 28(18):132-137 (in Chinese) 